\title{
Ein übereinzelsprachliches kontrastives Beschreibungsmodell für Partikelbedeutungen*
}

\author{
Gabriele Diewald (Hannover)/Marijana Kresić (Zadar)
}

\begin{abstract}
In this paper, a method for the semantic description of modal particles is presented. The method takes into account the context-sensitive meaning of this word class and offers an adequate basis for the purpose of language learning. Modal particles are analysed as grammatical markers of spoken dialogical language use. The model developed here can serve to describe the meaning of German modal particles as well as for contrastive semantic descriptions of particles. The contrastive application is demonstrated using the example of the language pair German-Croatian. First, an overview of the inventory and of the grammatical features of the word class of modal particles in German and of their Croatian equivalents is given. Then, existing methods of determining particle meanings are discussed. On the basis of the scheme for the description of particle meanings proposed by Diewald (1997, 2007), which relates to the Natural Semantic Metalanguage developed by Wierzbicka (e.g. 1996), a new universal model for the description of particle meanings is proposed. This new model is demonstrated with reference to the German modal particles doch, denn and ja and their Croatian equivalents $p a, m a$ und $a$. Finally, some remarks on the use of the model for the purpose of learning German as a foreign language are made.
\end{abstract}

\section{$1 \quad$ Einleitung}

Ziel dieses Beitrags ist es, ein Verfahren der Bedeutungsbeschreibung für die Modalpartikeln vorzustellen, das die kontextbezogene, dialoggrammatische Grundbedeutung dieser Wortklasse berücksichtigt und für die Zwecke des Fremdsprachenlernens eine geeignete Grundlage bietet. Das hier vorgestellte Vorhaben ist Teil eines kooperativen Projekts zur kontrastiven Beschreibung der Funktion und Semantik der Modalpartikeln, das von der Leibniz Universität Hannover (Germanistische und Angewandte Sprachwissenschaft, Gabriele Diewald) und der Universität Zadar (Institut für Linguistik, Marijana Kresić) durchgeführt wird. Mit dem hier entwickelten Modell lässt sich die Bedeutung der deutschen Modalpartikeln beschreiben, zugleich ist es für sprachvergleichende semantische Beschreibung von Partikeln einsetzbar. Die kontrastive Anwendung wird an dem Sprachenpaar Deutsch-Kroatisch vorgeführt.

Zunächst wird ein Überblick über das Inventar und über grammatische Merkmale der Wortklasse der Modalpartikeln im Deutschen und über ihre Entsprechungen im Kroatischen gegeben. Anschließend werden kurz vorliegende Ansätze zur Bestimmung von Partikelbedeutungen diskutiert. In Anlehnung an das von Diewald (1997, 2007) vorgeschlagene Schema zur Erfassung der Modalpartikelbedeutungen, das auf die von Wierzbicka (z. B. 1996) entwickelte Natural Semantic Metalanguage rekurriert, wird schließlich ein neues, übereinzelsprachliches Beschreibungsmodell vorgeschlagen und anhand der deutschen Modalpartikeln doch, denn und ja sowie ihrer kroatischen Entsprechungen $p a$, ma und $a$ demonstriert. Es schließen sich Überlegungen zur Didaktisierbarkeit und zum Einsatz des Beschreibungsmodells für das Erlernen des Deutschen als Fremdsprache sowie ein abschließendes Fazit an.

\footnotetext{
* This publication is based on work financed by the National Foundation for Science, Higher Education and Technological Development of the Republic of Croatia (project leader: Marijana Kresić). Partially supported by the Belgian Science Policy, Interuniversity Attraction Poles programme project P6/44, Gramis Project (project leader: Gabriele Diewald).
} 


\section{Deutsche Modalpartikeln und ihre kroatischen Entsprechungen: Inventar und grammatische Merkmale}

\subsection{Erste Bestimmung}

Das Deutsche verfügt über die formal und funktional distinkte Wortklasse der Modalpartikeln (vgl. z. B. Gelhaus 1998: 85-407; Diewald 2007). Im Kroatischen gibt es ebenfalls eine Klasse von Partikeln, die in Bezug auf Form, Funktion und Semantik weitgehend die gleichen Eigenschaften aufweisen, hinsichtlich ihrer Position im Satz aber variabel sind. Sowohl die deutschen Modalpartikeln als auch ihre kroatischen Entsprechungen sind unflektierbar, kommen hauptsächlich in gesprochener Sprache vor, modalisieren die durch den jeweiligen Satz ausgedrückte Proposition (in noch näher zu bestimmender Weise), sind Synsemantika, sind nicht negierbar, nicht koordinierbar, i. d. R. unbetont, syntaktisch weglassbar, dialoggrammatisch und pragmatisch jedoch unverzichtbar. Die deutschen Modalpartikeln gehören topologisch dem Mittelfeld an, die kroatischen Äquivalente sind dagegen vorfeldfähig.

Relevant für die semantische Beschreibung dieser Wortklasse sind außerdem zwei semantische Eigenschaften, die an dieser Stelle hervorzuheben sind: Die Partikeln weisen einerseits in beiden untersuchten Sprachen Heteroseme ${ }^{1}$ in anderen Wortklassen (z. B. dt. denn, kroat. $a$ : Konjunktion und Modalpartikel) auf und andererseits haben einzelne Partikellexeme verschiedene Bedeutungsvarianten.

\subsection{Inventare und Verwendungsbeispiele deutscher und kroatischer Modalpartikeln}

Folgende Mitglieder machen den Lexembestand der deutschen Modalpartikeln aus (nach Gelhaus 1998: 379): aber, auch, bloß, denn, doch, eben, eigentlich, etwa, halt, ja, mal, nur, schon, vielleicht, wohl. Als periphere Mitglieder sind zu nennen: fein, ganz, gerade, gleich, einfach, erst, ruhig (vgl. Diewald 2007: 118).

Das Kroatische verfügt über eine größere Anzahl an Partikeln. So zählt Uvanović (2006: 6676) 31 Partikellexeme zum Inventar der kroatischen Modalpartikeln: $a$, al (a), ama, bar(em), baš, da, de/hajdeldaj/dajte; e, eto, i, jednom, jednostavno, kako, li, ma, malo, moguće, možda, naprosto, onda, ono, pa, pak, samo, slobodno, što (li), valjda, već (jednom), više, zapravo, zar.

Hier sind zwei typische Beispiele für die Verwendung der Modalpartikeln im Deutschen (1) und Kroatischen (2) mit der jeweiligen Übersetzung in Klammern.

(1) Wie sind Sie denn darauf gekommen? ${ }^{2}$ [A kako ste se toga sjetili?] ${ }^{3}$

(2) Pa kako je biti letterer?

[Wie ist es denn Texter zu sein? $]^{4}$

\footnotetext{
${ }^{1}$ Hier wird im Anschluss an Autenrieth (2002: 3), Meibauer (1994) und andere der Begriff der Heterosemie verwendet, um die semantische Beziehung zwischen Modalpartikeln und identischen Wortformen in anderen Wortklassen zu bezeichnen. Heterosemie liegt im Fall historischer Verwandtschaft zwischen zwei oder mehr Bedeutungen bzw. Funktionen vor, d. h. dass diese auf eine gemeinsame Quelle zurückzuführen sind, sich jedoch im Laufe der Grammatikalisierung zu verschiedenen Wortklassen in unterschiedlichen morphosyntaktischen Kategorien entwickelt haben. Helbig (1988) z. B. bezeichnet diese Bedeutungsbeziehung als Homonymie, wogegen jedoch die gemeinsame Kernbedeutung der betreffenden Lexeme und ihre etymologische Verwandtschaft spricht.

${ }^{2}$ Auszug aus einem Beratungsgespräch beim Arbeitsamt (Becker-Mrotzek/Fickermann 1994: 97).

${ }^{3}$ Die kroatischen Übersetzungen der deutschen Beispiele stammen von M. Kresić.

${ }^{4}$ Auszug aus einem Interview mit einem Comic-Zeichner (http://www.zid.hr/Strip\%20-\%20Robert\%20Solanovic.htm, Stand 01.02.2009).
} 


\section{3 denn, doch, ja und ihre Entsprechungen im Kroatischen}

Auf der Grundlage vorliegender Arbeiten (Engel/Mrazović 1986: 1412-1454; Uvanović 2006: 66-76) ergeben sich für die von uns näher betrachteten Partikeln folgende Entsprechungen:

$$
\begin{aligned}
& \mathrm{dt} .: \text { denn - kroat.: ama, a, i, kako, ma, pa, ta, zar } \\
& \mathrm{dt} .: \text { doch - kroat.: a, bar, e pa, i, ma, pa, ta, ono, samo } \\
& \mathrm{dt} .: \text { ja - kroat.: e, eto, baš, pa, ta, samo }
\end{aligned}
$$

Es wird deutlich, dass einer deutschen Modalpartikel mehrere kroatische Partikellexeme entsprechen ${ }^{5}$. Bei den kontrastiven Analysen auf der Grundlage des hier vorgestellten Beschreibungsmodells (Kap. 4.3) erfolgt eine Beschränkung auf die hochfrequenten und polysemen kroatischen Partikeln $m a, p a$ und $a$.

Die Verwendung der einzelnen Modalpartikellexeme und ihrer Bedeutungsvarianten ist im Kroatischen ebenso wie im Deutschen auf bestimmte Strukturtypen bzw. Sprechakte verteilt. Zwar weist das Kroatische insgesamt eine größere Anzahl an Partikellexemen auf, in einigen Kontexten werden jedoch anstelle einer Modalpartikel andere sprachliche Mittel zum Zwecke der Abtönung bzw. Kontextualisierung von Äußerungen eingesetzt. Eine besondere Rolle spielen hier verschiedene syntaktische Mittel und die Intonation, d. h. es liegen keine 1:1-Entsprechungen vor.

\subsection{Grammatischer Status der Modalpartikeln}

Im Gegensatz zu Positionen, wonach Modalpartikeln "Synsemantika ohne grammatische [...] Funktionen" (Hentschel/Weydt 2002: 647) sind, stufen wir Modalpartikeln als grammatische Elemente ein (Diewald 2006: 405, 2007: 128; vgl. auch Autenrieth 2002: 236; Wegener 2002: 379).

Wie in Diewald/Kresić/Smirnova (2009) erörtert, erfüllt die Wortkategorie der Modalpartikeln zwei Kriterien, die für die Einordnung als grammatische Kategorie maßgeblich sind: Erstens weisen die Modalpartikeln das indexikalische Potenzial eines grammatischen Zeichens auf, das sich auf seinen spezifischen relationalen Inhalt bezieht (vgl. Diewald 2006: 414) und zweitens zeigen sie als Klasse die typische paradigmatische Integration grammatischer Kategorien (ebd.).

Die Funktion der Wortklasse der Modalpartikeln ist eine dialoggrammatische: Eine Modalpartikel markiert die Äußerung, in der sie enthalten ist, als nicht-initial. Dieses wird erreicht, indem die Proposition durch die Modalpartikel auf eine pragmatisch vorgegebene Einheit bezogen wird, wodurch die partikelhaltige Äußerung als reaktiver Zug in einer unterstellten dialogischen Sequenz markiert wird. Als wichtiger Punkt bei dieser Bestimmung ist festzuhalten, dass die Markierung einer Äußerung als nicht-initial durch eine Modalpartikel keineswegs bedeutet, dass partikelhaltige Äußerungen nicht als initiale Gesprächsschritte vorkommen dürften. Beispiele wie denn in Was hätten Sie denn gern? oder mal in Darf ich Sie mal was fragen?, die auch als gesprächseinleitende Äußerungen denkbar sind, zeigen ja eindeutig, dass partikelhaltige Äußerungen nicht von initialen Zügen ausgeschlossen sind. Jedoch wird aufgrund der Partikelbedeutung, die einen Verweis auf einen pragmatischen Prätext enthält, ein Diskurszusammenhang "behauptet" bzw. "hergestellt". Dieser kann real gegeben sein (z. B. durch vorgängige Gesprächsschritte), er muss aber nicht gegeben sein. Im letzten Fall ist die partikelhaltige Außerung faktisch initial und die reaktive Anbindung wird durch sie lediglich konstruiert bzw. behauptet. Wie andere grammatische Elemente können also auch die Modalpartikeln verwendet werden, um eine vom Sprecher erwünschte Perspektive zu erzeugen. Als Vergleich sei die Verwendung des Tempus "Präsens" im sogenannten historischen Präsens herangezogen: obwohl der dargestellte Sachverhalt anerkannter-

\footnotetext{
5 Entgegen der von manchen Autor/innen geäußerten Behauptung, dass das Kroatische bzw. Serbokroatische "wesentlich weniger Abtönungspartikeln kennt" (Engel/Mrazović 1986: 1412) als das Deutsche, vertreten wir die Auffassung, dass das Kroatische sogar eine größere Anzahl an Partikellexemen aufweist (s. o.), deren genaue paradigmatische Organisation im Vergleich zum Deutschen noch zu erforschen ist.
} 
maßen nicht mit dem Sprechzeitpunkt konzentrisch ist, wird durch die Verwendung des Präsens die Perspektive der Gleichzeitigkeit von Sachverhalt und Sprechzeit simuliert. Analoges geschieht bei der Verwendung von Modalpartikeln in initialen Äußerungen. Dass dies möglich ist, ist ein starkes Indiz für ihre reaktive semantische Komponente.

Auf welche Weise der Sprecher jeweils auf einen als pragmatisch vorgegeben Kontext referiert, variiert von Modalpartikellexem zu Modalpartikellexem, so dass hier die paradigmatische Organisation der Wortklasse zum Tragen kommt. Die einzelnen Partikellexeme nehmen je spezifische Positionen in einem höchst strukturierten, noch zu erforschenden grammatischen Paradigma ein. Da der Wechsel von initialen und responsiven bzw. reaktiven Turns konstitutives Merkmal mündlicher Kommunikation ist, handelt es sich bei der relationalen Funktion der Modalpartikeln um ein unentbehrliches grammatisches Mittel zur Dialogstrukturierung (vgl. Diewald 2006).

\section{$3 \quad$ Ansätze zur Bedeutungsbeschreibung der Modalpartikeln}

\subsection{Bedeutungsminimalismus vs. Bedeutungsmaximalismus}

Die zahlreichen Arbeiten zur Semantik der Modalpartikeln und die Vielzahl der darin präsentierten Lösungen können hier in keiner Weise gewürdigt werden. Verwiesen sei hierzu auf eine Darstellung in Diewald (2007). Für den hier verfolgten Zweck sind bezüglich der Modalpartikelsemantik vor allem zwei Aspekte relevant, die nach wie vor kontrovers diskutiert werden (vgl. Brünjes 2008: 27):

(1) Wie viele Bedeutungen sind einer Modalpartikel zuzuweisen?

(2) Welches ist die Klassenbedeutung der Modalpartikeln?

Diese Fragen werden auf zweierlei Weise beantwortet: durch bedeutungsmaximalistische und durch bedeutungsminimalistische Ansätze. Bedeutungsmaximalistische Ansätze zeichnen sich dadurch aus, dass sie

lexikalischen Elementen eine ganze Reihe (mehr oder minder kontextabhängiger) Bedeutungen zuordnen, ohne eine Reduktion auf interagierende Module und damit die Ermittlung einer Grundbedeutung überhaupt anzustreben. (Meibauer 1994: 4)

Für jede Verwendungsvariante einer Modalpartikel wird demnach eine eigene Bedeutung angenommen. Bedeutungsmaximalistisch verfahren beispielsweise Helbig (1988) und Helbig/ Kötz (1981). Der Grundgedanke bedeutungsminimalistischer Ansätze hingegen besteht darin,

für ein gegebenes lexikalisches Element möglichst wenige Bedeutungen anzunehmen, und andere Bedeutungseffekte auf nichtsemantische Komponenten oder auf die Interaktion von Komponenten zurückzuführen. (Meibauer 1994: 3)

Minimalistische Ansätze stellen eine Ableitungsbeziehung zwischen Bedeutungsvarianten oder einer Modalpartikel und ihren Heterosemen her. Dies kann etwa durch die Untersuchung historischer Zusammenhänge geschehen (z. B. Hentschel 1986). Ein anderes Mittel ist die explizite Formulierung einer Grundbedeutung, die allen Varianten gemeinsam ist. Die Entstehung der Varianten wird durch das Zusammenspiel von Grundbedeutung und kontextuellen Faktoren erklärt. Schon Weydt (1969: 35), aber auch andere Autor/innen gehen von dieser Annahme aus. Auch wir vertreten in unserer Analyse und den im Folgenden vorgestellten Modellen einen bedeutungsminimalistischen Ansatz, indem wir in der Bedeutungsbeschreibung von der allen Modalpartikeln gemeinsamen Klassenbedeutung ausgehen, dass sie die Äußerung, in der sie enthalten sind, auf den pragmatischen Kontext bzw. auf eine i. d. R. nicht verbalisierte Vorannahme beziehen.

Der Vorteil bedeutungsmaximalistischer Ansätze wiederum besteht darin, dass sie den Facettenreichtum der Bedeutungen auch einzelner Partikelvarianten im Detail erfassen können. In unserem kontrastiven Beschreibungsmodell kombinieren wir daher die bedeutungsminimalistische Betonung einer Grundbedeutung mit der Annahme von Partikelvarianten in verschiedenen Satzmodi. Im Folgenden soll kurz auf Helbig (1988/1994) als einschlägiges Beispiel für eine derartige bedeutungsmaximalistische Vorgehensweise eingegangen werden. 


\subsection{Helbigs Partikellexikon}

Helbigs Partikellexikon (1988: 31) enthält eine Beschreibung der Funktion und Distribution verschiedener Partikelklassen, darunter der Abtönungspartikeln. Es ist sowohl für den praxisorientierten Anwender konzipiert als auch für linguistische Zwecke. Exemplarisch sei hier der Eintrag für das Partikellexem ja in Helbigs Lexikon näher betrachtet.

$\mathrm{ja}_{4} \quad$ (Abtönungspartikel)

1. in Entscheidungsfragen; betont

2. Nur in Verbindung mit auch; Sprecher hebt hervor, wie wichtig die Richtigkeit des erfragten (und durch auch bereits abgetönten) Sachverhalts für ihn ist, betont sie zusätzlich und erwartet positive Antwort.

3. Hast du auch já den Schlüssel nicht vergessen?

Hast du auch já richtig zugehört?

Bist du auch já vorsichtig gefahren?

Abbildung 1: Ein Eintrag zur Modalpartikel ja aus Helbig (1988), s. V.ja

Wie aus Abbildung 1 ersichtlich, setzen die Einträge pro Strukturtyp bzw. Satzart (z. B. Aussagesatz, Entscheidungsfrage) ein eigenes Partikellemma an (hier ja).

Die Stärke des Partikellexikons von Helbig (1988) liegt ohne Zweifel darin, dass es alle Partikelvarianten - sowohl innerhalb der Subklasse der Modalpartikeln als auch in anderen Partikelklassen - in ihrer jeweils spezifischen Bedeutung und Verwendungsweise erfasst. Dies ist in dem Anspruch begründet, ein allgemeines Partikellexikon vorzulegen, das alle Partikeln der deutschen Sprache in all ihren Verwendungsvarianten einbezieht. Die Bedeutungsbeschreibung ist daher maximal differenzierend. Allerdings lässt sich in der Bedeutungsbeschreibung keine Systematik erkennen, so dass eine hinreichende Benutzeroder gar Lernerfreundlichkeit nur bedingt gewährleistet ist. Zu kritisieren ist zudem, dass es sich bei den angeführten Beispielen nicht um authentische Sprachbelege handelt, sondern um zum Teil konstruiert wirkende, isolierte Einzelsätze, die den für die Beschreibung von Partikelbedeutungen so wichtigen Kontext nicht berücksichtigen.

Das hier vorgeschlagene lernerfreundliche, kontrastive Beschreibungsmodell für Partikelbedeutungen kombiniert die bedeutungsminimalistische Zuweisung einer allen Modalpartikeln gemeinsamen Grundbedeutung mit der fein differenzierten Unterscheidung einzelner Modalpartikelvarianten auf der Grundlage ihrer Verteilung auf bestimmte Strukturtypen, wie sie auch von Helbig (1988) vorgenommen wird.

\subsection{Zur kontrastiven Bedeutungsbeschreibung kroatischer Modalpartikeln}

Im Gegensatz zu der Fülle an - methodisch und theoretisch recht unterschiedlich ausgerichteten - Untersuchungen zu den deutschen Modalpartikeln, steht die Erforschung der kroatischen Partikeln noch relativ am Anfang. Im Folgenden werden einige der zentralen Arbeiten in ihren Grundzügen skizziert.

Mit Ličen/Dahl (1981) liegt eine Untersuchung zum Serbokroatischen vor, die sprachvergleichend zum Deutschen angelegt ist. Dahl (1988) bietet eine Beschreibung der Funktion und Bedeutung deutscher Modalpartikeln auf der Grundlage der Sprechakttheorie; die Arbeit umfasst auch eine Liste der entsprechenden serbokroatischen Partikeläquivalente. Dabei wird die modalisierende Funktion der Partikeln vor allem in dem Ausdruck von Sprechereinstellungen gesehen. Engel/Mrazović (1986: 1412-1454) listen für die deutschen Modalpartikeln alle serbokroatischen Übersetzungsäquivalente auf, und zwar unter Abgrenzung von Heterosemen und unter Verweis auf andere modalisierende sprachliche Mittel des Serbokroatischen. Uvanović (1997) ist eine grundlegende kontrastive Studie zu den Modalpartikeln im Deutschen und Kroatischen, wobei die kroatischen Äquivalente zu den deutschen Modalpartikeln aber, denn, doch und schon auf der Grundlage eines Korpus literarischer Übersetzungen ermittelt werden. Uvanović (2006) liefert eine umfassende kontrastive, von spezifischen Sprechabsichten ausgehende Analyse kroatischer Partikeln bzw. Konversations- 
marker ${ }^{6}$, wobei die jeweiligen Entsprechungen im Deutschen und im Englischen ermittelt werden.

Insgesamt zeigt sich, dass in den grundlegenden Arbeiten zur kroatischen Grammatik und zu den Wortarten des Kroatischen ${ }^{7}$ Partikeln meist als unflektierbare Synsemantika (z. B. Silić/Pranjković 2007: 253) bzw. als Wörter mit bestimmten grammatischen Funktionen (z. B. Roguž 1997: 277) definiert werden. Die weitere Subklassifizierung der Partikeln erfolgt selten systematisch, wobei von keinem der Autoren eine zum Deutschen analoge Wortkategorie der Modalpartikeln angenommen wird.

Bei Sesar (1992: 253f.) etwa wird eine spezielle Klasse der Modalpartikeln für das Kroatische nicht angesetzt, sondern es werden alle kroatischen Partikelklassen als modale Ausdrucksmittel charakterisiert. Den Partikeln wird auf semantischer Ebene die allgemeine Funktion zugewiesen, Einstellungen, Eindrücke, den Willen und Wunsch des Sprechers auszudrücken (ebd.: 258). Barić et al. (2005) führen Partikeln zwar als distinkte Wortkategorie ${ }^{8}$ auf, spezifizieren diese jedoch nicht weiter hinsichtlich morphologischer und syntaktischer Kriterien, sondern bieten eine Subklassifikation auf der Grundlage verschiedener Sprecherintentionen an. Spezifisch für das Kroatische ist die Existenz von Fragepartikeln wie zar und $l i$, worauf u. a. Babić et al. (2007) hinweisen. Darauf bezieht sich auch ihre Anmerkung (ebd.: 571), dass Partikeln als unflektierbare Wortart der Modellierung der Satzstruktur bzw. des Strukturtyps dienen können. Auch die der Satznegation dienende Negationspartikel ne zählen z. B. Babić et al. (2007: 572) zu der letztgenannten Untergruppe. Als weitere Funktionen der Partikeln werden (ebd.: 571) die Hervorhebung oder Bedeutungsveränderung einzelner Wörter sowie die subjektiv-modale Bewertung des gesamten Satzes genannt. Den in der vorliegenden Arbeit vorgestellten Modalpartikeln entsprechen lediglich die in $\S 1201$ (ebd.: 572) u. a. aufgeführten Partikeln bar, $b a \check{s}, m a$, $t a$, die in allgemeiner Weise als Mittel der Hervorhebung definiert werden (ebd.). Die Grammatik von Težak/Babić (2000: 165f.) erwähnt Modalpartikeln in dem für das Deutsche definierten Sinne überhaupt nicht, sondern geht nur von einer Oberkategorie der Partikeln aus, die verschiedene satzstrukturmodellierende und bedeutungsfokussierende Funktionen haben. Als Ausgangspunkt für weitere kontrastive Analysen der Modalpartikeln geeignet ist die in der "Praktischen kroatischen Grammatik" von Raguž (1997: 277-286) gebotene Auflistung kroatischer Partikellexeme, die sowohl syntaktische als auch semantische Merkmale enthält, jedoch ebenfalls ohne eine eigene Klasse der Modalpartikeln.

Demgegenüber wird in kontrastiven Studien zum Kroatischen und zum Deutschen von einer Existenz der Klasse der Modalpartikeln in der kroatischen Sprache ausgegangen, was aus dem semantisch-funktionalen Vergleich mit dem Deutschen abgeleitet wird, z. B. bei den bereits erwähnten Autoren Engel/Mrazović (1986: 1412-1454) und Uvanović (2006).

Das "Kontrastive Beschreibungsmodell für Partikelbedeutungen" lässt sich in die Linie der letztgenannten Gruppe von Arbeiten einordnen, indem es die Existenz der Modalpartikeln in verschiedenen Sprachen durch morphosyntaktische Kriterien, vor allem aber durch das gemeinsame "Tertium Comparationis", ihre dialoggrammatische Funktion begründet. Diese gemeinsame grammatische Funktion bzw. Grundbedeutung wird im nachfolgenden Abschnitt näher bestimmt. Die hier vertretene These ist, dass sowohl das Deutsche als auch das Kroatische über eine funktional äquivalente Wortklasse "Modalpartikeln" verfügen. Die beiden gemeinsame Funktion besteht darin, dass sie die jeweilige Äußerung, in der sie enthalten sind, in systematischer Weise auf einen pragmatisch vorgegebenen Kontext beziehen. In der Forschungsliteratur zum Kroatischen ist eine auf diese Weise definierte Wortklasse nicht vertreten.

\footnotetext{
6 Die kroatischen Partikeln bezeichnet Uvanović (2006: 27) als Konversationsmarker. Durch diese terminologische Festlegung entsteht die Möglichkeit der Verwechslung mit der Wortkategorie der Diskurspartikeln.

${ }^{7}$ Bei diesem kurzen Forschungsüberblick werden hauptsächlich Arbeiten zum Kroatischen und einige frühere Arbeiten zum Serbokroatischen berücksichtigt. Ein Vergleich mit dem in jüngerer Zeit meist getrennt untersuchten Serbischen bleibt einer weiteren Studie vorbehalten.

${ }^{8}$ Partikeln werden von Barić et al. (1997: 282) als "čestice", "riječce" oder "partikule" bezeichnet.
} 
Zudem bietet keine der vorliegenden Arbeiten ein systematisches kontrastives Modell für die Bedeutungsbeschreibung der Partikeln, das zugleich für Lern- und Lehrzwecke einsetzbar ist. Diese Lücke soll mit dem hier vorgestellten Projekt geschlossen werden.

\section{$4 \quad$ Ein übereinzelsprachliches kontrastives Beschreibungsmodell für Partikelbedeutungen}

Wie in früheren Arbeiten erörtert (vgl. z. B. Diewald 1997: 77-79; Diewald 2007; Diewald/ Kresić/Smirnova 2009), besteht die Grundbedeutung der Modalpartikeln in dem bereits erwähnten Verweis (vgl. Kap. 2.4) auf eine pragmatisch vorgegebene Einheit, d.h. in der relationalen Verweisstruktur, die durch die Modalpartikel zwischen partikelhaltiger Proposition und einem angenommenen pragmatischen Kontext hergestellt wird. Ihre damit verbundene Grundfunktion ist es, wie auch Weydt (2001: 789) meint, "die Äußerung im univers du discours zu verankern, Bezüge zu Ko- und Kontext herzustellen". Diese Grundfunktion, die auch für die kroatischen Partikeln angenommen wird, kann mit folgendem Beschreibungsinstrumentarium expliziert werden.

\subsection{Dreizeiliges Notationsschema nach Diewald (2006, 2007 u. a.) und Wierzbickas (1996 u. a.) Natural Semantic Metalanguage (NSM)}

Ein auf den pragmatischen Kontext verweisendes, dreizeiliges Notationsschema zur Beschreibung von Partikelbedeutungen schlägt Diewald (z. B. 2007; vgl. auch Diewald/Fischer 1998) vor. Die erste Zeile formuliert die pragmatisch präsupponierte Einheit - also mindestens eine Variante der in der Äußerung dargestellten Proposition sowie - je nach Partikel präsupponierte Spechakttypen oder weitere Faktoren, die als gegeben unterstellt werden. Die zweite Zeile stellt die relevante Situation - also den aktuellen kommunikativen Zug - dar. Die dritte Zeile gibt die in der Situation tatsächlich getätigte Äußerung wieder: also die partikelhaltige sprachliche Einheit.

Das Schema soll in seiner Anordnung die "rückbezügliche Komponente" der Modalpartikelfunktion andeuten. Daher steht die partikelhaltige Äußerungen in der letzten, der dritten Zeile: d.h. sie ergibt sich aus der "Verrechnung" der präsupponierten Einheit mit der relevanten Situation.

$\begin{array}{ll}\text { pragmatisch präsupponierte Einheit } & \begin{array}{l}\text { Variante der Proposition p', } \\ \text { (Sprechakttyp s') } \\ \text { (weitere Faktoren) }\end{array} \\ \text { relevante Situation } & \begin{array}{l}\text { Proposition p } \\ \text { \& Sprechakttyp s }\end{array} \\ \text { partikelhaltige Äußerung } & \begin{array}{l}\text { Proposition p, } \\ \text { Sprechakttyp s } \\ \text { \& Abtönungspartikel }\end{array}\end{array}$

Tabelle 1: Basisschema zur Bedeutungsbeschreibung von Abtönungspartikeln (Diewald 2007: 134)

Wierzbickas Natural Semantic Metalanguage. Als semantische Beschreibungssprache wird auf die Natural Semantic Metalanguage (NSM) verwiesen, wie sie von Wierzbicka und ihren Mitarbeiten seit nunmehr einigen Jahrzehnten entwickelt wird (Wierzbicka 1986, 1991, 1996). Die universale semantische Metasprache definiert eine kleine Gruppe natürlich-sprachlicher Ausdrücke als semantische Primitive (z. B. dem Englischen entnommene Formen wie I, you, say, think, and etc.), mit deren Hilfe Wortbedeutungen ebenso wie ihre spezifischen Gebrauchsbedingungen notiert werden können. Durch diese Art der Bedeutungsparaphrase ist es möglich, illokutive und situative Komponenten systematisch und einheitlich zu notieren. Dies ist eine notwendige Bedingung für die angemessene Beschreibung von Modalpartikeln, die ja keine referenzielle Bedeutung haben. Außerdem erlaubt dieser Typus der semantischen Metasprache eine konsistente und gleichrangige Darstellung der nichtreferenziellen und der referenziellen Bedeutung. 


\subsection{Ein lernerorientiertes, kontrastives Beschreibungsmodell für Partikel- bedeutungen}

Auf der Grundlage der Natural Semantic Metalanguage (NSM) hat Brünjes (2008) in Anlehnung an das dreizeilige Notationsschema von Diewald $(2006,2007)$ ein Instrument zur Bedeutungsbeschreibung der Modalpartikeln entwickelt. Das Instrument beinhaltet den Verweis auf die relevante Situation bzw. den jeweiligen pragmatischen Prätext, der durch die modalpartikelhaltige Äußerung hergestellt wird. Die Bedeutungsbeschreibung erfolgt mit Hilfe der semantischen Primitive und einiger Leerstellen bzw. Variablen (z. B. p für Proposition). Die so entwickelten Schemata und insbesondere die NSM-basierten Bedeutungserklärungen ermöglichen eine für linguistisch-theoretische Zwecke adäquate und exakte Beschreibung der Kontextabhängigkeit von Partikelbedeutungen.

Für angewandte, d. h. didaktische und lexikografische Zwecke bedürfen diese Modelle jedoch einer Vereinfachung. Im Rahmen des hier verfolgten Ansatzes wird daher zur Beschreibung der lexemspezifischen Bedeutungen und der Art des Verweises auf den pragmatischen Vortext eine Art lexikografisches Minimalvokabular verwendet, das für das Erlernen des Deutschen als Fremdsprache ein leichter verständliches und memorierbares Beschreibungsformat ermöglicht.

Das "Zwei-Felder-Schema" zur Beschreibung von Partikelbedeutungen formalisiert und vereinfacht das Grundkonzept von Diewald (2006, 2007) und Brünjes (2008) so weit, dass es für die Zwecke des Fremdsprachenlernens eine geeignete Grundlage bietet. Ausgangspunkt ist wie bei Helbig (1988) die Zuordnung der Partikellexeme zu bestimmten Strukturtypen. Es wird hier allerdings - wie schon ausgeführt - ein bedeutungsminimalistischer Ansatz verfolgt, der auf die Festlegung einer Grundbedeutung für jede Partikel abzielt.

\begin{tabular}{|l|l|l|}
\hline & $\begin{array}{l}\text { lexemspezifischer } \\
\text { Kontextbezug }\end{array}$ & xy Verweis auf Gedanken / Kontext \\
\cline { 2 - 3 } & $\begin{array}{l}\text { relevante Situation } \\
=\text { Sprecher/in denkt: }\end{array}$ & XY: Satz \\
\hline $\begin{array}{l}\text { Grundbedeutung MPs } \\
\text { = Sprecher/in sagt: }\end{array}$ & MP-Äußerung $\leftarrow$ nicht am Anfang der Kommunikation \\
\hline
\end{tabular}

Das Beschreibungsschema: Grundbedeutung der Modalpartikeln

Mit Hilfe dieses von unten nach oben zu lesenden Zwei-Felder-Schemas wird in systematischer Weise auf den pragmatischen Kontext von partikelhaltigen Äußerungen Bezug genommen. In dem grau unterlegten, unteren Feld wird die konkrete, partikelhaltige Außerung eingetragen. Darüber ist die allen Modalpartikeln gemeinsame Grundbedeutung angegeben. Diese besteht darin, die betreffende Äußerung als nicht-initial zu markieren (s. a. Kap. 2.4). Das darüber liegende, weiße Feld enthält den vom Sprechenden für relevant gesetzten, eigenen mentalen Inhalt (Sprecher/in denkt: xy im Grundschema), der dann durch die Spezifizierung von XY in seiner Art genau bestimmt werden kann. In der obersten Zeile steht diese abstrakte Angabe, und zwar die Angabe, welcher Art der mit der Modalpartikel hergestellte Verweis auf den pragmatischen Kontext bzw. auf die Sprecher- und/oder Hörerkognition ist (bei ja z. B. affirmativ).

Auf diese Weise lassen sich unter konstantem Verweis auf die allen Modalpartikeln gemeinsame Grundbedeutung in spezifischen schematischen Beschreibungen die Bedeutungsvarianten für die einzelnen Partikellexeme erfassen, was im Folgenden am Beispiel von ja, denn und doch sowie der kroatischen Entsprechungen $a$, ma und pa vorgeführt wird. ${ }^{9}$

\footnotetext{
${ }^{9}$ Bei den partikelhaltigen Sätzen handelt es sich um authentische Korpusbelege.
} 
Beschreibungsmodell für Partikelbedeutungen

\section{a) Beschreibung der Bedeutung der deutschen Modalpartikeln ja, denn und doch}

\begin{tabular}{|l|l|l|}
\hline & $\begin{array}{l}\text { lexemspezifischer } \\
\text { Kontextbezug }\end{array}$ & $\begin{array}{l}\text { affirmativer Verweis auf (unterstellten) gemeinsamen } \\
\text { Gedanken }\end{array}$ \\
\cline { 2 - 3 }$=$ Sprecher/in denkt: & $\begin{array}{l}\text { Ich weiß und du weißt: Is leider immer noch so, dass } \\
\text { Elektroberufe so überwiegend Männerberufe sind. }{ }^{10}\end{array}$ \\
\cline { 2 - 3 } & Grundbedeutung MPs & MPßerung $\leftarrow$ nicht am Anfang der Kommunikation \\
\cline { 2 - 3 } & Sprecher/in sagt: & $\begin{array}{l}\text { Is ja leider immer noch so, dass Elektroberufe so } \\
\text { überwiegend Männerberufe sind. }\end{array}$ \\
\hline
\end{tabular}

ja (im Aussagesatz)

Das im Aussagesatz vorkommende ja markiert, wie alle Modalpartikeln, die Äußerung, in der es vorkommt, als nicht-initial. Gleichzeitig verweist der Sprecher durch die Verwendung der Partikel ja in dem von ihm geäußerten Aussagesatz affirmierend auf einen im pragmatischen Prätext angesiedelten, d.h. im Raum stehenden, gemeinsamen Gedanken, d. h. auf ein mit dem Hörer geteiltes Vorwissen. Der Sprecher markiert, dass beiden, ihm und dem Hörer, der Inhalt der durch den Satz ausgedrückten Proposition bereits bekannt ist. Bezogen auf den Hörer handelt es sich dabei um eine Unterstellung, die nicht zutreffen muss (s. a. Kap. 2.4).

\begin{tabular}{|l|l|l|}
\hline & $\begin{array}{l}\text { lexemspezifischer } \\
\text { Kontextbezug }\end{array}$ & Verweis auf Faktoren, die die Frage motivieren \\
\cline { 2 - 3 }$=$ Sprecher/in denkt: & $\begin{array}{l}\text { Aufgrund dessen, was vorher war, frage ich: Welches } \\
\text { Buch war das? }\end{array}$ \\
\cline { 2 - 3 } & Grundbedeutung MPs & MP-Äußerung $\leftarrow$ nicht am Anfang der Kommunikation \\
\cline { 2 - 3 } & $=$ Sprecher/in sagt: & Welches Buch war denn das?
\end{tabular}

\section{denn (im Fragesatz)}

Mit denn markiert der Sprecher die von ihm geäußerte Frage als nicht-initial. Die Partikel denn fungiert als Indikator dafür, dass der Sprecher im pragmatischen Kontext Faktoren ansetzt, die seine Frage motivieren. Aufgrund nicht näher spezifizierter, vorausgehender Kontextfaktoren richtet der Hörer die betreffende Frage an den Sprecher.

\begin{tabular}{|l|l|l|}
\hline & $\begin{array}{l}\text { lexemspezifischer } \\
\text { Kontextbezug }\end{array}$ & $\begin{array}{l}\text { Verweis auf Rückkehr zu zwischenzeitlich verneinter } \\
\text { Aussage }\end{array}$ \\
\cline { 2 - 3 }$=$ Sprecher/in denkt: & $\begin{array}{l}\text { Richtig ist: Das ist nicht die Bibel. } \\
\text { Möglich wäre: Das ist die Bibel. }\end{array}$ \\
\cline { 2 - 3 } & Grundbedeutung MPs & MP-Äußerung $\leftarrow$ nicht am Anfang der Kommunikation \\
\cline { 2 - 3 }$=$ Sprecher/in sagt: & Das ist doch nicht die Bibel.
\end{tabular}

doch (im Aussagesatz)

Das Auftreten der Modalpartikel doch im Aussagesatz markiert diesen gleichfalls als nicht am Beginn des kommunikativen Austausches angesiedelt. Durch die Verwendung von doch

10 Der Beispielsatz entstammt dem Korpus "Beratungen beim Arbeitsamt" (Becker-Mrotzek/Fickermann (1994: 98).

11 Als Grundbedeutung der Modalpartikel doch in Aussagesätzen wird die Indizierung einer Auswahl aus zwei Alternativen angenommen (vgl. Diewald 1997: 90f. und Diewald/Fischer 1998: 92f.). Es wird einer im Raum stehenden Alternative bzw. Verneinung (vgl. auch Sekiguchi 1977) der betreffenden Proposition widersprochen, d. h. der Hinweis auf den pragmatischen Kontext hat Widerspruchscharakter. 
verweist der Sprecher auf einen nur aus dem pragmatischen Kontext rekonstruierbaren, nicht versprachlichten Dreischritt:

\section{Proposition, 2. verneinte Proposition, 3. Proposition + doch .}

Mit doch wird also die Rückkehr zu einer zwischenzeitlich verneinten Aussage markiert. Die in dem partikelhaltigen Satz ausgedrückte Proposition wird als ursprüngliche und richtige angesehen, welche zwischenzeitlich oder potenziell verneint wurde. Der angeführte Beispielsatz zeigt, dass dies auch mit umgekehrten Vorzeichen erfolgen kann, indem die Negation im Zwischenschritt aufgehoben wird.

\section{b) Sprachkontrastive Modalpartikelbeschreibungen kroatisch-deutsch: ja - pa, denn - a,} doch - ma

\begin{tabular}{|l|l|l|}
\hline & $\begin{array}{l}\text { lexemspezifischer } \\
\text { Kontextbezug }\end{array}$ & $\begin{array}{l}\text { affirmativer Verweis auf (unterstellten) gemeinsamen } \\
\text { Gedanken }\end{array}$ \\
\cline { 2 - 3 }$=$ Sprecher/in denkt: & $\begin{array}{l}\text { Ich weiß und du weißt: Is leider immer noch so, dass } \\
\text { Elektroberufe so überwiegend Männerberufe sind. }{ }^{12} \\
\text { Nažalost je još uvijek tako da su zanimanja na području } \\
\text { elektrike pretežno muška zanimanja. } \text { [Übersetzung] }\end{array}$ \\
\cline { 2 - 3 } & Grundbedeutung MPs & $\begin{array}{l}\text { MP jaßerung } \leftarrow \text { nicht am Anfang der Kommunikation } \\
\text { überwiegend Männer noch so, dass Elektroberufe so sind. } \\
\text { Pa nažalost je još uvijek tako da su zanimanja na } \\
\text { području elektrike pretežno muška zanimanja. }\end{array}$ \\
\hline
\end{tabular}

ja (deutsch) - pa (kroatisch) (im Aussagesatz)

Die Übersetzung des oben bereits analysierten deutschen Aussagesatzes mit ja im Aussagesatz zeigt, dass dem kroatischen Übersetzungsäquivalent $p a$ die gleiche Funktion wie dem deutschen ja zukommt. Beide markieren die Äußerung, in der sie vorkommen, als nichtinitial. Gleichzeitig verweist der Sprecher sowohl durch die Verwendung der Partikel ja als auch durch den Gebrauch von $p a$ in dem entsprechenden kroatischen Satz affirmierend auf einen im pragmatischen Prätext angesiedelten, d.h. im Raum stehenden, gemeinsamen Gedanken, auf ein mit dem Hörer geteiltes Vorwissen. Der Sprecher markiert, dass beiden, ihm und dem Hörer, der Inhalt der durch den Satz ausgedrückten Proposition bereits bekannt ist (s. o.).

\begin{tabular}{|l|l|l|}
\hline & $\begin{array}{l}\text { lexemspezifischer } \\
\text { Kontextbezug }\end{array}$ & $\begin{array}{l}\text { affirmativer Verweis auf (unterstellten) gemeinsamen } \\
\text { Gedanken }\end{array}$ \\
\cline { 2 - 3 }$=$ Sprecher/in denkt: & $\begin{array}{l}\text { Snimke su } i \text { na YouTube- } u \text {. [Die Aufnahmen sind auch } \\
\text { auf YouTube.] }\end{array}$ \\
\cline { 2 - 3 } & Grundbedeutung MPs & MP-Äußerung $\leftarrow$ nicht am Anfang der Kommunikation \\
\hline$=$ Sprecher/in sagt: & $\begin{array}{l}\text { Pa snimke su } i \text { na YouTube- } u^{13} \text { [Die Aufnahmen sind ja } \\
\text { auch auf YouTube.] }\end{array}$ \\
\hline
\end{tabular}

$p a$ (kroatisch) - ja (deutsch) (im Aussagesatz)

Speisen wir nun anstelle der Übersetzung einen authentischen kroatischen Satz, in dem die Partikel $p a$ vorkommt, in das kontrastive Beschreibungsmodell ein, zeigt sich, dass der

12 Der Beispielsatz entstammt dem Korpus "Beratungen beim Arbeitsamt" (Becker-Mrotzek/Fickermann (1994: 98).

13 Authentischer Beleg aus einem Fernsehinterview

(http://dnevnik.hr/vijesti/hrvatska/bolid-nad-hrvatskom.html, Stand 20.01.2009). 
affirmative Verweis auf einen (unterstellten) gemeinsamen Gedanken auf die gleiche Weise vorgenommen wird.

\begin{tabular}{|l|l|l|}
\hline & $\begin{array}{l}\text { lexemspezifischer } \\
\text { Kontextbezug }\end{array}$ & Verweis auf Faktoren, die die Frage motivieren \\
\cline { 2 - 3 }$=$ Sprecher/in denkt: & $\begin{array}{l}\text { Aufgrund dessen, was vorher war, frage ich: Koliko } \\
\text { košta sloboda? [Was kostet die Freiheit?] }\end{array}$ \\
\cline { 2 - 3 } & Grundbedeutung MPs & MP-Äußerung $\leftarrow$ nicht am Anfang der Kommunikation \\
\cline { 2 - 3 }$=$ Sprecher/in sagt: & $\underline{\text { A koliko košta sloboda? [Was kostet denn die Freiheit?] }}$ \\
\hline
\end{tabular}

$a$ (kroatisch) - denn (deutsch) (im Fragesatz)

Wie die Analyse eines kroatischsprachigen Belegs mit $a$ zeigt, hat diese Partikel die gleiche Grundbedeutung und die gleiche spezifische Funktion wie die deutsche Modalpartikel denn: Auch mit $a$ markiert der Sprecher die von ihm geäußerte Frage als nicht-initial. $A$ fungiert als Indikator dafür, dass der Sprecher im pragmatischen Kontext Faktoren ansetzt, aus denen seine Frage abgeleitet ist. Sie ist durch nicht näher spezifizierte, vorausgehende Kontextfaktoren motiviert.

\begin{tabular}{|l|l|l|}
\hline & $\begin{array}{l}\text { lexemspezifischer } \\
\text { Kontextbezug }\end{array}$ & $\begin{array}{l}\text { Verweis auf Rückkehr zu zwischenzeitlich verneinter } \\
\text { Aussage }\end{array}$ \\
\cline { 2 - 3 }$=$ Sprecher/in denkt: & $\begin{array}{l}\text { Ich will: Pustit ćeš me. [Du lässt mich.] } \\
\text { Möglich wäre: Nećeš me pustiti. [Du lässt mich nicht.] }\end{array}$ \\
\cline { 2 - 3 } & Grundbedeutung MPs & MP-Äußerung $\leftarrow$ nicht am Anfang der Kommunikation \\
\cline { 2 - 3 } & $=$ Sprecher/in sagt: & Ma pusti me! [Lass mich doch!]
\end{tabular}

ma (kroatisch) - doch (deutsch) (im Aufforderungssatz)

Das kroatische ma markiert wie die deutsche Modalpartikel doch den betreffenden Aussagesatz als nicht-intial. Auch durch die Verwendung von $m a$ verweist der Sprecher auf den oben beschriebenen, im pragmatischen Prätext angesiedelten Dreischritt: 1. Proposition, 2. verneinte Proposition, 3. Proposition $+m a$. D. h. mit $m a$ wird ebenso wie mit doch die Rückkehr zu einer zwischenzeitlich verneinten Aussage markiert.

Anhand der kontrastiven Bedeutungserklärung der deutschen Modalpartikeln ja, denn und doch und ihrer kroatischen Entsprechungen $p a, a$ und $m a$ konnte gezeigt werden, dass für beide Sprachen eine geteilte Grundbedeutung sowie eine gleiche Art des spezifischen Verweises auf den jeweiligen pragmatischen Prätext angenommen werden kann.

\section{Lernerorientierte Konzeption des Beschreibungsmodells}

Das Beschreibungsformat des Zwei-Felder-Schemas ist so konzipiert, dass es dem Anspruch der Lernerorientierung bzw. Lernerfreundlichkeit gerecht wird. Das Erfüllen der folgenden Kriterien wird hierfür als maßgeblich angesehen:

Verständlichkeit der Erklärungen: Es wird ein Minimalvokabular bzw. ein MiniDefinitionswortschatz für die Beschreibung der Partikelbedeutungen verwendet, das - bis auf wenige gesondert erläuterte Fremdwörter - für Lernende ab dem Mittelstufenniveau verständlich ist. Dieses Minimalvokabular ist in Anlehnung an analoge lexikografische Minimalwortschätze formuliert (z. B. Oxford Advanced Learner's Dictionary 2000: 1529-1537); an seiner genauen Ausgestaltung wird derzeit noch gearbeitet. Zum Definitionswortschatz gehören Wörter wie Sprecher, Hörer, sagen, denken u. a.m. Da es sich bei der zu beschreibenden Wortkategorie um Funktionswörter handelt mit zum Teil abstrakten grammatischen und pragmatischen Bedeutungskomponenten, ist die Hinzunahme ausgewählter, spezifischer Fachwörter und Internationalismen unerlässlich (z. B. Kontext, affirmativ u. a.). Zwar können auch diese Fachtermini aufgelöst, d. h. in Paraphrasen umgeformt werden, doch 
bläht diese das Beschreibungsformat ohne zusätzlichen Gewinn auf. Hier wird noch nach lernerfreundlichen Lösungen gesucht. Eine Möglichkeit besteht darin, ein aus wenigen Einträgen bestehendes Fachvokabluar einleitend zu erläutern und kontrolliert anzuwenden.

Einfachheit und Memorierbarkeit der Partikelbeschreibungen: Die Modalpartikelbedeutungen werden mit Hilfe eines transparenten und universellen Zwei-Felder-Schemas beschrieben, das die kontextbezogene Semantik der Modalpartikeln so formalisiert, dass sie für DaF-Lernende nachvollziehbar und im Rahmen des DaF-Unterrichts vermittelbar wird.

Übertragbarkeit/Anwendbarkeit auf andere Modalpartikeln und Sprachen: Es gibt ein Grundschema, das die gemeinsame Bedeutung der Modalpartikeln beschreibt, und spezifische Varianten für jede einzelne Partikel. Sowohl das Basisschema als auch die spezifischen Partikelvarianten können auf partikelhaltige Äußerungen aus anderen Sprachen angewandt bzw. mit diesen kontrastiert werden. Zudem kann sich der Lernende mit Hilfe des Beschreibungsschemas die Bedeutung konkreter partikelhaltiger Äußerungen bzw. der betreffenden Modalpartikeln selbst erschließen, indem er den jeweiligen Satz in das Schema für die jeweils spezifische Modalpartikel einspeist.

\section{$6 \quad$ Fazit}

Wir hoffen, gezeigt zu haben, dass die Annahme einer dialoggrammatischen Funktion der Klasse der Modalpartikeln und der darauf aufbauenden distinktiven Bedeutungen der Einzellexeme eine sinnvolle Generalisierung darstellt. Diese grammatische Funktion bildet den gemeinsamen Nenner der in der gesprochenen Sprache hochfrequenten und typischen Partikelklasse. Zudem ergibt sich aus dieser Perspektive, d. h. aus der Interpretation der Modalpartikeln als (dialog-)grammatische Kategorie, die Möglichkeit zu einer einfachen Beschreibung der distinktiven Bedeutungen der Einzellexeme, die natürlich jeweils nur die distinktive Kernbedeutung benennt und somit unterdeterminiert bezüglich stärker differenzierender Varianten ist.

Ferner haben wir gezeigt, dass das gewählte Beschreibungsschema kontextuelle Faktoren in kontrollierter Weise einbezieht, so dass bei Bedarf Polysemien und kontextabhängige Partikellesarten einheitlich dargestellt werden können. Das Beschreibungsschema könnte auch an Funktionen und Kontextfaktoren und deren Serialisierung in der Kommunikation ausgerichtet werden und also onomasiologisch vorgehen. Zugleich ist es die Grundlage für sprachvergleichende und für den Fremdsprachenunterricht didaktisierbare Beschreibungsmodelle. Dies haben wir zunächst an zwei Partikelsprachen gezeigt.

Im Rahmen des hier vorgestellten Projekts werden schematisierte Beschreibungen für alle eingangs inventarisierten Modalpartikellexeme des Deutschen und des Kroatischen entwickelt. Die Annahme der Wortkategorie der Modalpartikeln auch für das Kroatische lässt sich, wie die durchgeführten Analysen gezeigt haben, aus der für diese Wortart charakteristischen, und auch für die kroatischen Partikeln typischen Grundbedeutung ableiten. Diese besteht darin, dass eine Modalpartikel 1. die jeweilige Äußerung als nicht-initial markiert und 2. in einer jeweils spezifischen Art und Weise auf einen pragmatischen Prätext bzw. auf einen vom Sprecher als relevant gesetzten, eigenen mentalen Inhalt referiert.

Das hier vorgeschlagene Beschreibungsformat bietet für die Wortklasse der Modalpartikeln ein lernerorientiertes, d.h. verstehbares, memorierbares und auf andere Fälle anwendbares Beschreibungsformat, das sich in sprachkontrastive Lehr- und Lernmaterialen transformieren lässt (vgl. dazu auch Kresić/Ferraresi 2006).

\section{Literatur}

Autenrieth, Tanja (2002): Heterosemie und Grammatikalisierung bei Modalpartikeln: eine synchrone und diachrone Studie anhand von "eben", "halt", "ech(er)t", "einfach", "schlicht" und "glatt". Tübingen: Niemeyer.

Babić, Stjepan et al. (2007): Glasovi i oblici hrvatskoga književnoga jezika. Zagreb: Nakladni zavod globus.

Barić, Eugenija et al. (2005): Hrvatska gramatika. 4. Aufl. Zagreb: Školska knjiga. 
Becker-Mrotzek, Michael/Fickermann, Ingeborg (1994): "Beratungen beim Arbeitsamt und im Versicherungsamt". In: Redder, Angelika/Ehlich, Konrad (eds.): Gesprochene Sprache. Transkripte und Tondokumente. Tübingen, Niemeyer: 91-135.

Brünjes, Lena (2008): Die Bedeutung deutscher Modalpartikeln. Entwicklung eines Beschreibungsinstrumentes auf der Grundlage der Natural Semantic Metalanguage. Leibniz Universität Hannover: Examensarbeit.

Dahl, Johannes (1988): Die Abtönungspartikeln im Deutschen: Ausdrucksmittel für Sprechereinstellungen mit einem kontrastiven Teil deutsch-serbokroatisch. Heidelberg: Groos.

Diewald, Gabriele (1997): Grammatikalisierung. Eine Einführung in Sein und Werden grammatischer Formen. Tübingen: Niemeyer (= Germanistische Arbeitshefte 36).

Diewald, Gabriele (2006): "Discourse Particles and Modal Particles as Grammatical Elements". In: Fischer, Kerstin (ed.): Approaches to Discourse Particles. Amsterdam, Elsevier: 403-426.

Diewald, Gabriele (2007): "Abtönungspartikel". In: Hoffmann, Ludger (ed.): Handbuch der deutschen Wortarten. Berlin, de Gruyter: 117-141.

Diewald, Gabriele/Kresić, Marijana/Smirnova, Elena (2009): "The grammaticalization channels of evidentials and modal particles in German: integration in textual structures as a common feature." In: Mosegaard Hansen, Maj-Britt/Visconti, Jacqueline (eds.): Diachronic Semantics and Pragmatics. Amsterdam, Elsevier: 193-209.

Diewald, Gabriele/Fischer, Kerstin (1998): "Zur diskursiven und modalen Funktion der Partikeln aber, auch, doch und ja in Instruktionsdialogen." Linguistica 38: 75-99.

Engel, Ulrich/Mrazović, Pavica (eds.) (1986): Kontrastive Grammatik Deutsch-Serbokroatisch. München: Otto Sagner.

Gelhaus, Hermann (1998): "Die Wortarten". In: Dudenredaktion (ed.): Duden. Grammatik der deutschen Gegenwartssprache. 6. Aufl. Mannheim, Duden: 85-407.

Helbig, Gerhard (1988): Lexikon deutscher Partikeln. 3. Aufl. Leipzig: Langenscheidt.

Helbig, Gerhard/Kötz, Werner (1981): Die Partikeln. Leipzig: Langenscheidt Verlag Enzyklopädie.

Hentschel, Elke (1986): Funktion und Geschichte deutscher Partikeln. Ja, doch, halt und eben. Tübingen: Niemeyer.

Hentschel, Elke/Weydt, Harald (2002): "Die Wortart 'Partikel'". In: Wiegand, Herbert Ernst (ed.): Handbücher zur Sprach- und Kommunikationswissenschaft. Bd. 21: Lexikologie. Berlin, de Gruyter. 646-653.

Kresić, Marijana/Ferraresi, Gisella (2006): "Transfer beim Erlernen des Deutschen als Fremdsprache. Das Beispiel Italienisch-Deutsch". Estudios Filológicos Alemanes. Vol. 11: 325342.

Ličen, Marina/Dahl, Johannes (1981): "Die Modalpartikeln ja und doch und ihre serbokroatischen Entsprechungen". In: Weydt, Harald (ed.): Partikeln und Deutschunterricht: Abtönungspartikeln für Lerner des Deutschen. Heidelberg, Groos: 213-223.

May, Corinna (2000): Die deutschen Modalpartikeln: wie übersetzt man sie (dargestellt am Beispiel von eigentlich, denn und überhaupt, wie lehrt man sie? Ein Beitrag zur kontrastiven Linguistik (deutsch-spanisch, spanisch-deutsch) und Deutsch als Fremdsprache. Frankfurt a. M. etc.: Lang.

Meibauer, Jörg (1994): Modaler Kontrast und konzeptuelle Verschiebung. Studien zur Syntax und Semantik deutscher Modalpartikeln. Tübingen: Niemeyer (= Linguistische Arbeiten 314).

Wehmeier, Sally (ed.) (2004). Oxford Advanced Learner's Dictionary of Current English. 6. Aufl. Oxford: Oxford University Press.

Roguž, Dragutin (1997): Praktična hrvatska gramatika. Zagreb: Medicinska naklada.

Sekiguchi, Tsugio (1977): "Was heißt doch?" In: Weydt, Harald (ed.): Aspekte der Modalpartikel: Studien zur deutschen Abtönung. Tübingen, Niemeyer: 3-9.

Sesar, Dubravka (1992): "O mogućnostima kategorizacije partikula u hrvatskom jezičnom standardu". Suvremena lingvistika, Svezak: 18-34.

Silić, Josip/Pranjkoyić, Ivo (2007): Gramatika hrvatskoga jezika za gimnazije $i$ visoka učilišta. Zagreb: Školska knjiga. 2. Aufl.

Težak, Stjepko/Babić, Stjepan (2004): Gramatika hrvatskoga jezika. Priručnik za osnovno jezično obrazovanje. Zagreb: Školska knjiga.

Uvanović, Željko (1997): Die deutschen Modalpartikeln aber, denn, doch und schon und ihre kroatischen Entsprechungen. Marburg: Tectum. 
Uvanović, Željko (2006): Kroatische Konversationsmarker. Versuch einer Extraktion im translatologischen Vergleich mit deutschen Modalpartikeln (d.h. Abtönungspartikeln) und deren englischen Entsprechungen. Norderstedt: books on demand.

Wegener, Heide (2002): "The Evolution of the German Modal Particle denn". In: Wischer, Ilse/Diewald, Gabriele (eds.): New Reflections on Grammaticalization. Amsterdam, Benjamins: $379-394$.

Weydt, Harald (1969): Abtönungspartikel. Bad Homburg v.d. H.: Verlag Gehlen (= Linguistica et Litteraria 4$)$.

Weydt, Harald (2001): "Partikelforschung". In: Holtus, Günther et al. (eds.): Lexikon der Romanistischen Linguistik. Bd. I, 1: Geschichte des Faches Romanistik; Methodologie (das Sprachsystem). Tübingen, Niemeyer: 782-801.

Wierzbicka, Anna (1986): "A Semantic Metalanguage for the Description and Comparison of Illocutionary Meanings". Journal of Pragmatics 10: 67-107.

Wierzbicka, Anna (1991): Cross-Cultural Pragmatics. The Semantics of Human Interaction. Berlin: Mouton der Gruyter.

Wierzbicka, Anna (1996): Semantics. Primes and Universals. Oxford: Oxford University Press.

\section{Online-Quellen/Korpora}

Berlin-Brandenburgische Akademie der Wissenschaften (ed.): Digitales Wörterbuch der deutschen Sprache des 20. Jahrhunderts (DWDS). www.dwds.de.

http://dnevnik.hr/vijesti/hrvatska/bolid-nad-hrvatskom.html

Stanić, Damjan: Intervju. Robert Solanovic. http://www.zid.hr/Strip\%20-\%20Robert\%20 Solanovic.htm. 